\title{
Magnetic Doppler imaging of early-type stars
}

\section{Oleg Kochukhov ${ }^{1}$, Thomas Rivinius ${ }^{2}$, Mary E. Oksala ${ }^{3}$ and Iosif Romanyuk ${ }^{4}$}

\author{
${ }^{1}$ Dept. Physics and Astronomy, Uppsala University, Box 516, 75120 Uppsala, Sweden \\ ${ }^{2}$ European Southern Observatory, Casilla 19001, Santiago 19, Chile \\ ${ }^{3}$ Dept. of Physics and Astronomy, University of Delaware, Newark, DE 19716, USA \\ ${ }^{4}$ Special Astrophysical Observatory of RAS, Nizhnij Arkhyz 369167, Russia
}

\begin{abstract}
Doppler imaging of early-type magnetic stars is the most advanced method to interpret their line profile variations. DI allows us to study directly a complex interplay between chemical spots, magnetic fields, and the mass loss. Here we outline the general principles of the surface mapping of stars, discuss adaption of this technique to early-type stars and present several recent examples of the abundance and magnetic mapping performed for rapidly rotating early-B stars. In particular, we present the first Doppler images for the very fast rotating He-rich star HR 7355 and a reconstruction of magnetic field for the well-known Bp star $\sigma$ Ori E. We also present new magnetic maps for the He-strong star HD 37776, which possesses one of the most complex magnetic field topologies among the upper main sequence stars.
\end{abstract}

Keywords. polarization, stars: chemically peculiar, stars: magnetic fields, stars: atmospheres, stars: individual (HR 7355, $\sigma$ Ori E, HD 37776)

\section{Introduction}

The presence of strong magnetic fields on the stellar surfaces leads to the formation of horizontal inhomogeneities, which manifest themselves in the periodic variation of brightness, flux distribution, and spectral line profiles. The late-type stars with convective envelopes possess small-scale, rapidly evolving magnetic fields, which are associated with dark cool spots. The geometry of these horizontal structures changes noticeably on the time-scales from days to months due to the emergence of new magnetic flux and stellar activity cycles. In these stars the dynamo mechanism responsible for the generation of magnetic fields is closely connected to the stellar rotation rate and differential rotation in the interior (Donati \& Landstreet 2009).

The hot stars with radiative envelopes exhibit spots of a different nature. A small fraction of A and B-type stars are slow rotators and show kG-strength magnetic fields on their surfaces. These fields appear to be stable and typically (but not always!) are dominated by a dipolar component (Landstreet 1992). The stabilizing effect of magnetic fields, the lack of the surface convection zone, and a slow rotation provide an ideal environment for the vertical segregation of chemical elements under the influence of atomic diffusion (Leblanc et al. 2009). Consequently, the magnetic A and B stars, also known as Ap/Bp stars, have a strikingly non-solar chemical composition of their atmospheres. Anisotropy of the diffusion processes introduced by the magnetic field also yields a strongly inhomogeneous distribution of chemical elements, both with height in the atmosphere (Kochukhov et al. 2006) and horizontally across the surface (Kochukhov et al. 2004). 

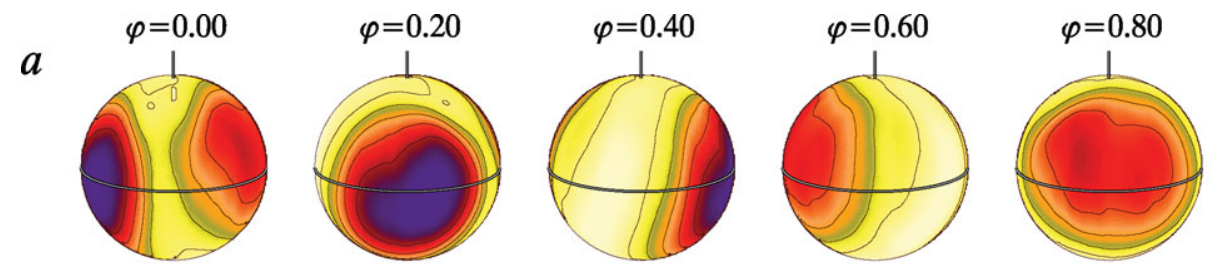

$\mathrm{B}(\mathrm{kG})$

$b$
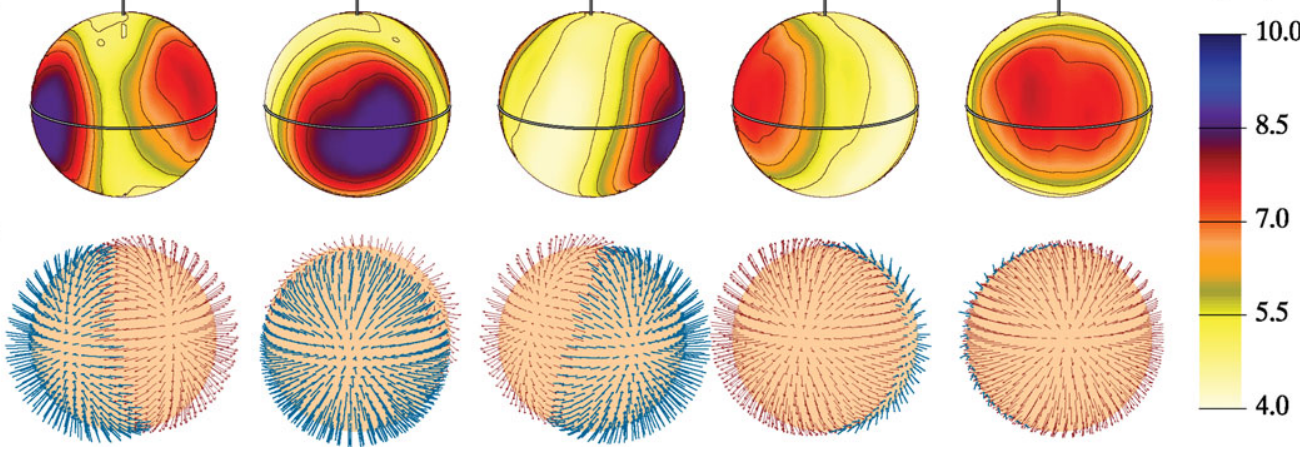

Figure 1. Surface magnetic field distribution reconstructed for $\sigma$ Ori E with magnetic Doppler imaging. The star is shown at five rotational phases as indicated above the spherical plots. The aspect corresponds to the inclination angle $i=75^{\circ}$ and vertically oriented rotational axis. a) The distribution of the magnetic field strength. The thick line shows the stellar rotational equator. Rotational axis is indicated by the short vertical bar. b) The orientation of the magnetic field vectors. In these vector maps the light arrows show the field vectors pointing outside the stellar surface and the dark arrows correspond to the vectors pointing inwards. The arrow length is proportional to the field strength.

Among the hottest and most massive stars, the Bp phenomenon is represented by the He-rich objects. These stars exhibit all of the phenomenology typical of the cooler magnetic Ap stars but, in addition to that, have strong radiately driven winds which interact with the global magnetic field, feeding an active and dynamic magnetosphere (Townsend et al. 2007). The properties of the magnetospheric material and its distribution around the star are directly related to the configuration and strength of the stellar magnetic field. Moreover, one has to understand and model in detail the stellar flux redistribution due to chemical spots in order to properly use the stellar brightness variation for the diagnosis of circumstellar environments. Thus, understanding the physical processes taking place at the surfaces and in the extended magnetospheres of early B-type stars requires one to develop a sophisticated surface models of the chemical star spots and magnetic fields.

In this contribution we review the progress in obtaining detailed chemical and magnetic maps of early-type stars with the help of the Doppler imaging (DI) technique and its recent extension to magnetic fields - magnetic DI.

\section{The principles of magnetic Doppler imaging}

Star spots leave a characteristic signature in the stellar line profiles. The spectrum of a spot usually differs substantially from the radiation from the rest of the stellar surface. Hence, the disk-integrated flux profile will contain a distortion - a dip (a deviation towards less flux) for chemical overabundance or a bump (a deviation towards more flux) for a cool spot - at the velocity shift corresponding to the spot's longitude on the stellar surface. As the star rotates, this distortion moves within the line profile, allowing to track individual spots. A numerical technique to reconstruct a two-dimensional map of the stellar surface from the line profile time-series is called Doppler imaging (DI).

DI has been successfully applied to mapping abundance and temperature spots on the stellar surfaces (Khokhlova et al. 1986; Vogt et al. 1987) and was extended to the problem of reconstructing vector magnetic field topologies (Brown et al. 1991; Piskunov \& Kochukhov 2002). Magnetic DI is considerably more challenging compared to its nonmagnetic counterpart due an increased number of degrees of freedom for vector maps 
and a typically poor signal-to-noise ratio of the stellar spectropolarimetric observations. Nevertheless, when all four Stokes parameter spectra with dense enough rotational phase coverage are available for the magnetic inversion, it is possible to obtain a unique solution without introducing an a priori information about the field geometry, such as multipolar parameterization (Kochukhov \& Piskunov 2002).

Different DI codes applied to the magnetic mapping of hot stars mainly differ in their treatment of the line formation and the degree of self-consistency in modeling magnetic fields and associated inhomogeneities. The mapping technique known as Zeeman Doppler imaging (e.g., Donati et al. 2006) uses a simple analytical description of the line shapes, limited to the weak-field regime and applied to a single average Stokes $V$ profile (LSD profile) without considering either chemical spots or linear polarization. Another family of codes (e.g., Kochukhov \& Wade 2010), used in the studies discussed below, models the polarized radiative transfer in detail for all four Stokes parameters and carries out a self-consistent mapping of spots and magnetic fields. The latter methodology is, however, much more demanding computationally and currently can be applied only to a few narrow spectral intervals at a time.

\section{Applications to early-type stars}

\section{1. $\sigma$ Ori $E$}

$\sigma$ Ori $\mathrm{E}$ (HD 37479) is a prototype He-strong star with magnetospheric structures and is by far the most studied object in its class. It exhibits synchronous modulation of brightness, UV resonance lines, and $\mathrm{H} \alpha$ emission with a period of $1.19 \mathrm{~d}$, all pointing to an accumulation of the circumstellar material in two corotating clouds situated at the intersection between the equator of a roughly dipolar magnetic field and rotational equator (Groote \& Hunger 1982). Considerable theoretical and multi-wavelength observational effort has been put forth in the past few years to develop a physically realistic model of the interaction between the magnetic field and the wind in this star (e.g., Townsend et al. 2005, this meeting). But despite the importance of $\sigma$ Ori $\mathrm{E}$ for our understanding of the hot-star magnetospheres, relatively little emphasis was put on collecting precise information about its magnetic field and the stellar surface structures. $\sigma$ Ori E is known to have $\sim 10 \mathrm{kG}$ dipolar-like magnetic field (Landstreet \& Borra 1978) and high-contrast surface abundance inhomogeneities (Reiners et al. 2000), yet the previous analyses of these surface structures did not proceed beyond simple parametric models of abundance spots (Veto et al. 1991) and dipolar fits to the longitudinal magnetic field measurements (Townsend et al. 2005). We have collected high-resolution, wide wavelength coverage circular polarization spectra of $\sigma$ Ori E with the ESPaDOnS and NARVAL polarimeters. These observations are interpreted with the DI code of Piskunov \& Kochukhov (2002) to obtain a new, more realistic and detailed model of the magnetic field geometry and study distribution of chemical spots. Fig. 1 shows preliminary magnetic field structure determined from the Stokes $I$ and $V$ profiles of the He I $6678 \AA$ line. Evidently, the magnetic field of $\sigma$ Ori $\mathrm{E}$ is approximately dipolar, in agreement with previous studies. However, magnetic DI map also reveals a considerable field strength difference between the two poles, suggesting the presence of a non-negligible quadrupolar component.

\subsection{HR 7355}

The unusual properties of HR 7355 (HD 182180) were pointed out by Rivinius et al. (2008), who found this star to be a very fast rotating emission-line object with an enhanced He absorption and variable spectral lines. With its rotation period of $0.521 \mathrm{~d}$ 


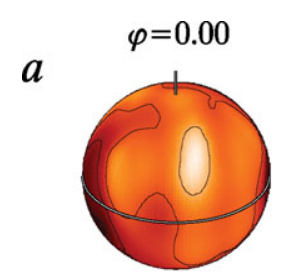

$b$
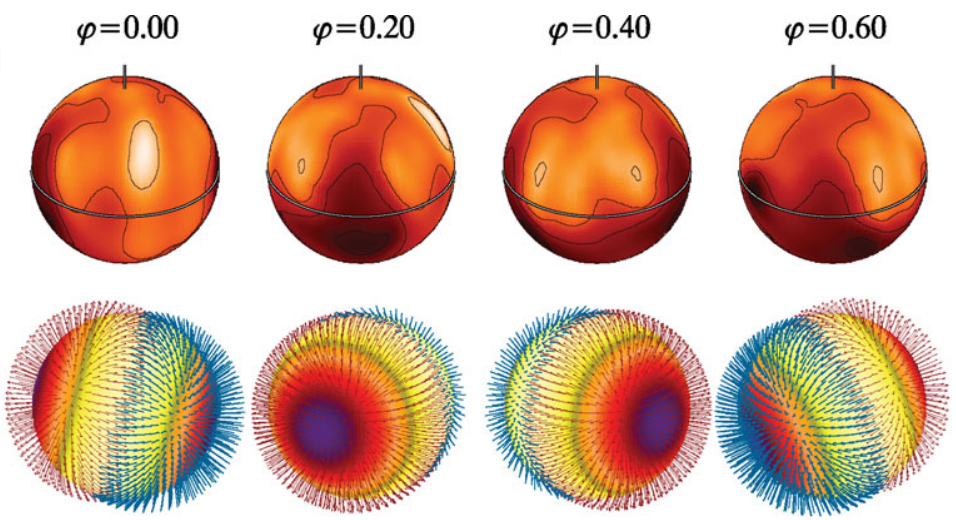

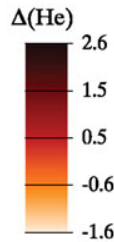

$\mathrm{B}(\mathrm{kG})$
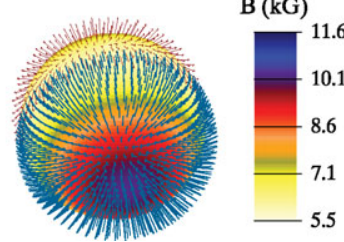

Figure 2. a) Doppler imaging map showing the He abundance distribution derived for HR 7355 . The He abundance is given on a logarithmic scale relative to the Sun. b) The dipolar magnetic field geometry adopted for HR 7355. This plot shows distribution of the magnetic field strength and orientation of the field vectors.

and $v_{\mathrm{e}} \sin i>300 \mathrm{kms}^{-1}$, HR 7355 stands out among Bp stars, which are generally expected to have slow to moderate rotation only. Recently, Oksala et al. (2010) and Rivinius et al. (2010) discovered a variable longitudinal magnetic field with the strength between -2.2 and $+3.2 \mathrm{kG}$. The roughly sinusoidal longitudinal field curve indicates a dipoledominated magnetic topology with a polar strength exceeding $10 \mathrm{kG}$. The presence of such an exceptionally strong magnetic field on a rapid rotator confirms the outstanding nature of HR 7355, pushing the boundaries of the Bp star phenomenon into a previously unexplored domain. Strong spectroscopic variability and rapid rotation make HR 7355 an ideal target for Doppler imaging. We are carrying out DI modeling of several absorption features in the UVES time-series spectra of this star with the aim to investigate the surface distributions of $\mathrm{He}, \mathrm{Si}$, and $\mathrm{C}$. The preliminary map of the He abundance obtained from the He I $4713 \AA$ line is presented in Fig. 2. This appears to be the first published Doppler image for such a rapidly rotating spotted star. The spectropolarimetric observations of HR 7355 do not yet have a phase coverage sufficient for magnetic DI. Therefore, our abundance mapping employed a dipolar field model with the parameters $B_{\mathrm{p}}=11.6 \mathrm{kG}$ and $\beta=78^{\circ}$ inferred from the longitudinal magnetic field observations. In the future we plan to perform simultaneous mapping of the magnetic field and chemical spots in this remarkable star.

\section{3. $H D$ 37rng}

HD 37776 (V901 Ori) is a B2IV He-strong star rotating with a period of $\approx 1.5387 \mathrm{~d}$. It shows variation of He and metal lines (Bohlender 1994), as well as a prominent rotational modulation of the brightness in different photometric bands (Adelman 1997). It is also one of the few spotted stars for which the rotational spin-down has been directly measured (Mikulášek et al. 2008) and the light variations were explained with a physically realistic star-spot model (Krtička et al. 2007). Uniquely for an early-type chemically peculiar star, HD 37776 shows a complex double-wave longitudinal magnetic field variation (Thompson \& Landstreet 1985), suggesting that its magnetic field topology significantly departs from an oblique dipolar field common for other Bp stars. Previous studies of the magnetic field structure of HD 37776 gave inconclusive results. Bohlender (1994) fitted the longitudinal field curve with an axisymmetric multipolar magnetic geometry, dominated by a quadrupolar component. Khokhlova et al. (2000) used a simplified version of the magnetic DI to derive a range of possible magnetic field geometries, consisting of a 

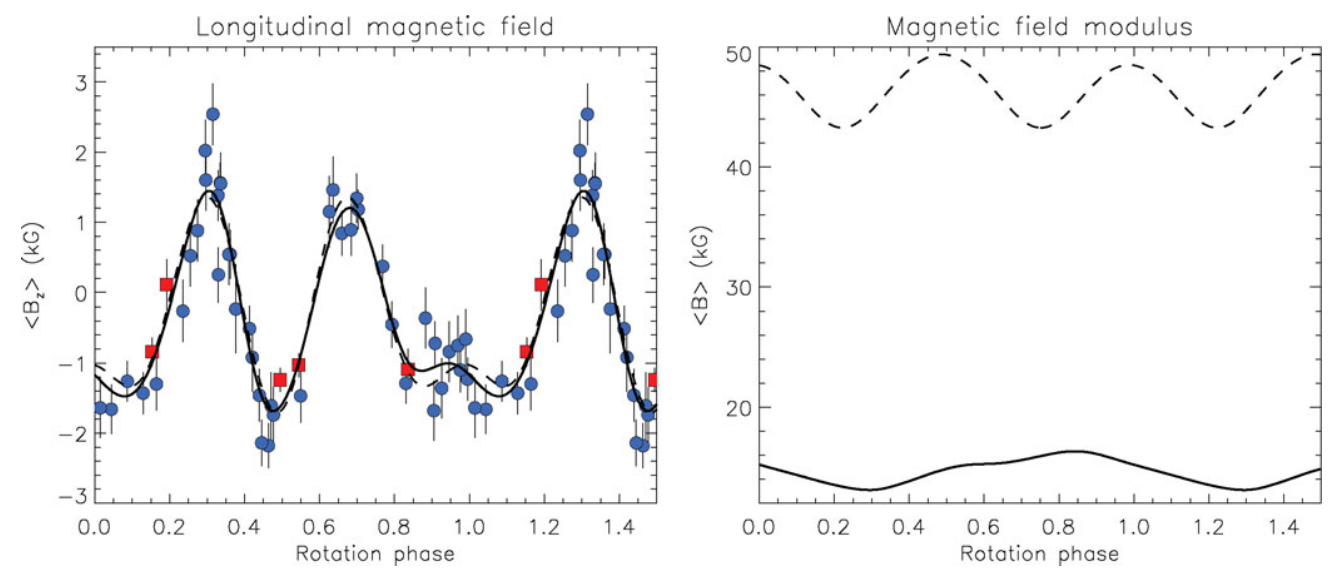

Figure 3. Left panel: comparison of the mean longitudinal magnetic field of HD 37776 measured by Thompson \& Landstreet (1985) (circles) and Donati et al. (1997) (squares) with the fit achieved by the axisymmetric multipolar model (dashed curve) and by the direct magnetic inversion (solid curve). Right panel: the mean field modulus variation predicted by the multipolar model (dashed curve) and by the magnetic DI field map (solid curve).

non-aligned dipole and quadrupole. None of the previous studies could find a magnetic field topology successfully reproducing both the longitudinal magnetic field measurements and the polarization in line profiles. At the same time, all studies agree that an exceptionally strong, $50-100 \mathrm{kG}$, non-dipolar field is needed to describe the magnetic observations of HD 37776 if the field topology is parameterized with low-order multipoles. If true, this would make HD 37776 the most magnetized non-degenerate star known. We carried out a new analysis of the magnetic field structure of HD 37776, combining the traditional fitting of the longitudinal magnetic field curve with a more sophisticated magnetic DI study of the circular polarization observations in the He I $5876 \AA$ line collected with the Russian 6-m telescope at the Special Astrophysical Observatory. Adopting an intermediate inclination angle $\left(i=50^{\circ}\right)$, we find that $\approx 100 \mathrm{kG}$ octupolar magnetic topology is required to describe the double-wave longitudinal field curve (Fig. 3). However, this multipolar model fails to match the circular polarization spectra, predicting Stokes $V$ profile of both a wrong amplitude and an incorrect shape for the significant fraction of the rotation cycle. This clearly illustrates the fundamental limitation of the magnetic modeling based on $\left\langle B_{\mathrm{z}}\right\rangle$ measurements alone.

To develop a new comprehensive magnetic field model of HD 37776, we combined interpretation of the longitudinal field measurements and radiative transfer modeling of the He I $5876 \AA$ line profiles in a single, self-consistent magnetic DI inversion, as was previously done for Ap stars (Kochukhov et al. 2002). Abandoning the assumption of a low-order multipolar field, we obtained a model of the magnetic field topology which for the first time successfully reproduced all magnetic observations available for HD 37776 . These DI results, illustrated in Fig. 4, suggest that the field geometry of this star is highly complex and non-axisymmetric, lacking any resemblance to a low-order multipolar structure. This field is, however, much weaker than suggested by the previous studies. The field strength does not exceed $\approx 30 \mathrm{kG}$ at the surface, yielding the phaseaveraged magnetic field modulus of only $\approx 14.5 \mathrm{kG}$. The new magnetic field topology of HD 37776 has important implications for understanding different aspects of the variability of this unique star and for our understanding of the early B-type star magnetism in general. 


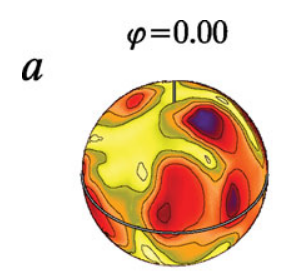

$b$

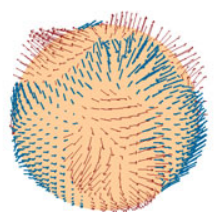

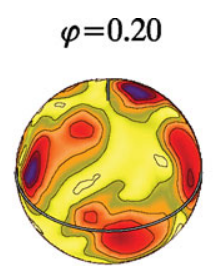
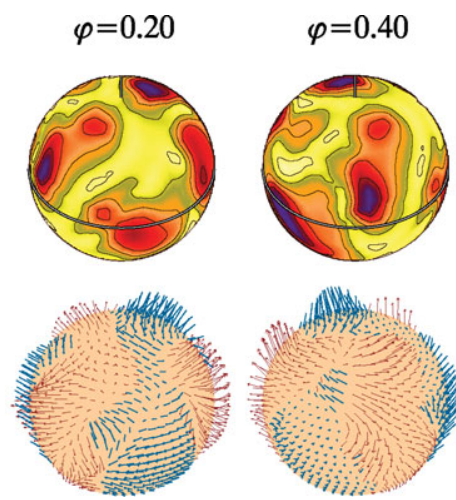

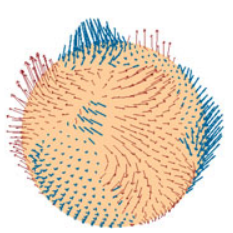

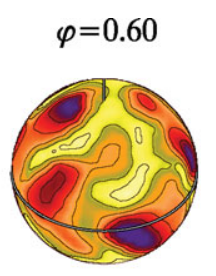
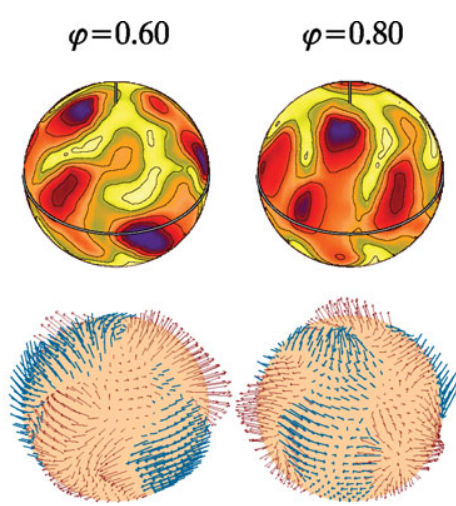

$\mathrm{B}(\mathrm{kG})$

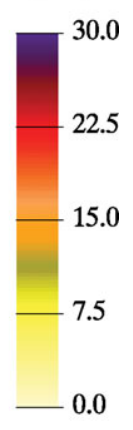

Figure 4. Surface magnetic field structure of HD 37776 derived with magnetic Doppler imaging. The format of this figure is similar to Fig. 1.

\section{Acknowledgements}

O.K. is a Royal Swedish Academy of Sciences Research Fellow supported by grants from the Knut and Alice Wallenberg Foundation and the Swedish Research Council.

\section{References}

Adelman, S. J. 1997, A\& $A S, 125,65$

Bohlender, D. A. 1994, in: L. A. Balona, H. F. Henrichs, \& J. M. Le Contel (eds.), Pulsation; Rotation; and Mass Loss in Early-Type Stars, IAU Symposium 162, p. 155

Brown, S. F., Donati, J.-F., Rees, D. E., \& Semel, M. 1991, A\&A, 250, 463

Donati, J.-F. \& Landstreet, J. D. 2009, ARAA, 47, 333

Donati, J.-F., Howarth, I. D., Jardine, M. M., Petit, P. et al. 2006, MNRAS, 370, 629

Donati, J.-F., Semel, M., Carter, B. D., Rees, D. E. et al. 1997, MNRAS, 291, 658

Groote, D. \& Hunger, K. 1982, A\&A, 116, 64

Khokhlova, V. L., Rice, J. B., \& Wehlau, W. H. 1986, ApJ, 307, 768

Khokhlova, V. L., Vasilchenko, D. V., Stepanov, V. V., \& Romanyuk, I. I. 2000, Astron. Lett., 26,177

Kochukhov, O., Drake, N. A., Piskunov, N., \& de la Reza, R. 2004, A\&SA, 424, 935

Kochukhov, O. \& Piskunov, N. 2002, A\&4, 388, 868

Kochukhov, O., Piskunov, N., Ilyin, I., Ilyina, S. et al. 2002, A\& $A, 389,420$

Kochukhov, O., Tsymbal, V., Ryabchikova, T., Makaganyk, V. et al. 2006, A\&A, 460, 831

Kochukhov, O. \& Wade, G. A. 2010, A\&A, 513, A13

Krtička, J., Mikuláček, Z., Zverko, J., \& Žižńovský, J. 2007, AESA, 470, 1089

Landstreet, J. D. 1992, A\&GAR, 4, 35

Landstreet, J. D. \& Borra, E. F. 1978, ApJ (Letters), 224, L5

Leblanc, F., Monin, D., Hui-Bon-Hoa, A., \& Hauschildt, P. H. 2009, A\&A, 495, 937

Mikulášek, Z., Krtička, J., Henry, G. W., Zverko, J. et al. 2008, A\&A, 485, 585

Oksala, M. E., Wade, G. A., Marcolino, W. L. F., Grunhut, J. et al. 2010, MNRAS, 405, L51

Piskunov, N. \& Kochukhov, O. 2002, A\&A, 381, 736

Reiners, A., Stahl, O., Wolf, B., Kaufer, A. et al. 2000, A\&AA, 363, 585

Rivinius, T., Szeifert, T., Barrera, L., Townsend, R. H. D. et al. 2010, MNRAS, 405, L46

Rivinius, T., Štefl, S., Townsend, R. H. D., \& Baade, D. 2008, A\& $A$, 482, 255

Thompson, I. B. \& Landstreet, J. D. 1985, ApJ (Letters), 289, L9

Townsend, R. H. D., Owocki, S. P., \& Groote, D. 2005, ApJ (Letters), 630, L81

Townsend, R. H. D., Owocki, S. P., \& Ud-Doula, A. 2007, MNRAS, 382, 139

Veto, B., Hempelmann, A., Schoeneich, W., \& Stahlberg, J. 1991, AN, 312, 133

Vogt, S. S., Penrod, G. D., \& Hatzes, A. P. 1987, ApJ, 321, 496 